Article

\title{
SUPERPOSITION OF MOTIONS IN THE SPACE OF LAGRANGE VARIABLES
}

\author{
Yu. A. Alyushin \\ National University of Science and Technology MISIS, Moscow, 119991, Russia \\ alyushin7@gmail.com; alyushin@misis.ru. Author ID in ORCID http://orcid.org/0000-0001-9977-3342. Tel.: + 79162218591
}

\begin{abstract}
The technique of superposition of motions in the space of Lagrange variables is described, which allows us to obtain the equations of combined motion by replacing the Lagrange variables of superimposed (external) motion with Euler variables of nested (internal) motion. The components of velocity and acceleration in the combined motion obtained as a result of differentiating the equations of motion in time coincide with the results of vector addition of the velocities and accelerations of the particles involved in the superimposed motions at each moment of time. Examples of motion and superposition descriptions for absolutely solid and deformable bodies with equations for the main kinematic characteristics of motion, including for robot manipulators with three independent drives, pressing with torsion, bending with tension, and cross- helical rolling, are given. Given the fragment of calculation of forces in the kinematic pairs shown the advantages of the description of motion in Lagrangian form for the dynamic analysis of lever mechanisms, allows to determine the required external exposure when performing the energy conservation law at any time in any part of the mechanism.
\end{abstract}

Keywords: continuous medium, equations of motion, Lagrange variables, superposition, dynamic analysis.

\section{Features of the description of motion in the form of Lagrange}

Any motion of a solid or deformable body can be described in the form of Lagrange $x_{i}=x_{i}\left(\alpha_{p}, t\right)$, Euler $\alpha_{p}=\alpha_{p}\left(x_{i}, t\right)$, or in a mixed way, for example, for displacement projections $u_{i}=u_{i}\left(x_{i}, \alpha_{p}, t\right)$. In continuum mechanics, Euler variables are usually used as arguments, explaining this by the comparative simplicity of the obtained relations. However, physical laws should be formulated not for points of space, but for material particles. From a physical point of view, Lagrange variables are more reasonable when describing motion [1] (p. 235), so the definition of functions

$$
x_{i}=x_{i}\left(\alpha_{p}, t\right),
$$

where $t$ is time, $x_{i} \in(x, y, z)$ and $\alpha_{p} \in(\alpha, \beta, \gamma)$ - the Euler and Lagrange variables, respectively, are considered the main problem of continuum mechanics [2] (p.23). However, the description of the movement in the form of Lagrange in the literature is given undeservedly little attention [3]. The advantages of Lagrange variables are associated with a fairly simple principle of superposition of motions [4], the possibility of determining a generalized measure of motion 
(energy) for each particle involved in motion, the use of the law of conservation of energy to predict the behavior of a mechanical system, the interpretation of the concept of "generalized force" through an increment of energy, which expands the possibilities of applying mechanics in solving various practical problems [5-6].

The aim of the work is to show the advantages of Lagrange variables and superposition in describing various types of motion and solving various problems of applied mechanics.

The different nature of the independent arguments of equations (1) leads to the need to use two differential operators for time $(d)$ and space $(\delta)$. The first characterizes the change of any property associated with motion for a particle over an infinitesimal period of time $d t$, for example coordinates $d x\left(\alpha_{p}, t\right)=x_{t}\left(\alpha_{p}, t\right) d t$ or functions $d f\left(\alpha_{p}, t\right)=f_{t}\left(\alpha_{p}, t\right) d t$. The second one defines increments of motion-related properties for adjacent particles whose Lagrangian coordinates in the initial state differed by infinitesimal values $\delta \alpha_{p} \in(\delta \alpha, \delta \beta, \delta \gamma)$, for example, the increment of the abscissa of adjacent particles is $\delta x=x_{\alpha} \delta \alpha+x_{\beta} \delta \beta+x_{\gamma} \delta \gamma$. To increment similar properties in time, two operators should be used simultaneously, for example, the change in time of the increment of the abscissa of adjacent particles is determined by the right side of the equation

$$
d \delta x=\delta d x=\left(x_{t \alpha} \delta \alpha+x_{t \beta} \delta \beta+x_{t \gamma} \delta \gamma\right) d t
$$

The independence of the two operators allows you to change the order of their entries without changing the final result.

Here and further, the lower indices in the designation of functions correspond to differentiation by the variables specified in the indices $\left(x_{t} \equiv d x / d t, x_{\alpha} \equiv \partial x / \partial \alpha\right)$. For index functions, for example coordinates $x_{i} \in x_{i}(x, y, z)$, the differentiation indices are indicated after the comma, for example $x_{i, t} \equiv d x_{i} / d t$ and $x_{i, \alpha} \equiv \partial x_{i} / \partial \alpha$. The initial coordinates of the points $\alpha=\left.x\right|_{t=0}, \beta=\left.y\right|_{t=0}, \gamma=\left.z\right|_{t=0}$ are taken as Lagrange variables.

In accordance with equations (1), in the general case, the motion is characterized by 12 first derivatives, including the components of the particle velocity $x_{i, t}$ and the components of the unsymmetric tensor of the second rank

$$
x_{i, p} \equiv \frac{\partial x_{i}}{\partial \alpha_{p}}=\left[\begin{array}{ccc}
x_{\alpha} & x_{\beta} & x_{\gamma} \\
y_{\alpha} & y_{\beta} & y_{\gamma} \\
z_{\alpha} & z_{\beta} & z_{\gamma}
\end{array}\right]
$$


which determines the rotation and deformation of the particle.

The sum of the squares of the derivatives in each column (with the same lower differentiation indices) is equal to the square of the ratio of the edge lengths of an infinitesimal parallelepiped in the current $\delta l$ and initial $\delta l_{0}$ states

$$
e_{p}^{2}=x_{p}^{2}+y_{p}^{2}+z_{p}^{2}=\left(\delta l / \delta l_{0}\right)^{2}, \quad p \in(\alpha, \beta, \gamma)
$$

The deformation of the particle is characterized by the average value

$$
e=\left(e_{\alpha}+e_{\beta}+e_{\gamma}\right) / 3
$$

and the standard deviation of the relative lengths of the edges (3) from their average value (4)

$$
\Gamma^{2}=\left(e_{\alpha}-e\right)^{2}+\left(e_{\beta}-e\right)^{2}+\left(e_{\gamma}-e\right)^{2}=\left(e_{p}-e\right)\left(e_{p}-e\right) .
$$

The quadratic invariant of the tensor (2)

$$
\Gamma_{e}^{2}=x_{i, p} x_{i, p}=x_{\alpha}^{2}+x_{\beta}^{2}+x_{\gamma}^{2}+y_{\alpha}^{2}+y_{\beta}^{2}+y_{\gamma}^{2}+z_{\alpha}^{2}+z_{\beta}^{2}+z_{\gamma}^{2}
$$

remains unchanged when the coordinate axes are rotated, is a generalized measure of elastic deformation [5] and can be represented as a sum

$$
\Gamma_{e}^{2}=3 e^{2}+\Gamma^{2}=x_{i, p} x_{i, p}=e_{p} e_{p}
$$

In the right-hand sides of the last two equations, the summation rule for the index repeated in the monomial is used.

The determinant of the matrix (2) or the Jacobian of the transformation (1) numerically coincides with the ratio of the particle volumes in the current $\delta V$ and initial $\delta V_{0}$ states

$$
R=\frac{\delta V}{\delta V_{0}}=\left|x_{i, p}\right|=\left|\begin{array}{ccc}
x_{\alpha} & x_{\beta} & x_{\gamma} \\
y_{\alpha} & y_{\beta} & y_{\gamma} \\
z_{\alpha} & z_{\beta} & z_{\gamma}
\end{array}\right| .
$$

If the values of $e_{p}$ are different from 1, the shape and / or volume of the particles change, the movement is accompanied by deformation. If

$$
e_{p}=1, \quad \quad R=1, \quad \quad e^{2}=3, \quad \Gamma=0,
$$

there is no deformation. Such cases correspond to the motion of absolutely solid bodies.

The body associated with the concept of "material point" differs from a real solid body the domain of the definition of Lagrange variables for which it is contracted to a point. However, this transition is not always appropriate. In particular, the analysis of the kinematics of rigid bodies with the actual scope of Lagrangian variables 
allows to account for the rotation of the body during its motion, to identify possible errors by the description of the equations of motion in the absence of strain and move by one form of description of motion to another.

\section{The principle of superposition}

In accordance with the generally accepted concepts [1-3], the superposition of motions is reduced to determining the velocity fields for each of the motions, summing them according to the rules of vector algebra, and then integrating the velocities over time to determine the position of particles in combined motion.

The final result can be obtained much easier if the combined movements are written in the Lagrange form. Then, in order to provide a vector addition of velocities at each moment of time, it is sufficient to replace the Lagrange variables of one motion with the Euler variables of another motion [4-5].

Formally, this replacement can be explained by a simple transition to new Lagrange variables, which take the coordinates of the particles at the current time. Motion can be considered as consisting of a number of stages, each of which can use either a new or a single frame of reference of the observer. In accordance with this, Lagrange variables can be introduced both independently at each stage, and for a sequence of several stages.

Consider two consecutive stages of arbitrary motion of a continuous medium on the time interval $0 \leq t \leq t_{2}$. Let the first stage be bounded by the time interval $0 \leq t \leq t_{1}<t_{2}$ for the equations of motion

$$
x_{i}=x_{i}\left(\alpha_{p}, t\right) \text { при } 0 \leq t \leq t_{1}<t_{2}
$$

where $\alpha_{p}$ are Lagrange variables, $\alpha_{i}=x_{i}$ at $t=0$. The Eulerian coordinates $x_{i}$ at the final moment of the considered stage at $t=t_{1}$ are distinguished by the notation

$$
\xi_{i}=x_{i}\left(\alpha_{p}, t_{1}\right)
$$

At the second stage $t_{1} \leq t \leq t_{2}$ you can enter a new time frame $t^{\prime}=t-t_{1} \geq 0$ and new Lagrange coordinates $\xi_{k}$ that coincide with the current coordinates (11) at time $t_{1}$ and allow you to describe the further process of movement in the form

$$
x_{i}=x_{i}\left(\xi_{k}, t^{\prime}\right) \text { when } 0 \leq t^{\prime} \leq t_{2}-t_{1}
$$

the time $t_{1}$ in the second stage should be considered as a constant. 
However, as Lagrange variables, you can take not only the initial coordinates, but also any functions of the initial coordinates that are uniquely related to them. In particular, relations (11) or equations of motion (10) can be chosen as such functions for $t=t_{1}$. Formally, in this case, equations (12) preserve the form

$$
x_{i}=x_{i}\left(\xi_{k}\left(\alpha_{p}\right), t^{\prime}\right) \quad \text { for } \quad 0 \leq t^{\prime} \leq t_{2}-t_{1}
$$

but the Lagrange variables should be considered as functions of the original variables $\alpha_{p}$ in accordance with equations (11).

On the other hand, equations (12) can be extended to the second stage without changing the time frame, it is enough only to limit the time interval of this stage in the original time scale

$$
x_{i}=x_{i}\left(\xi_{k}, t\right) \quad \text { at } \quad t_{1} \leq t \leq t_{2}
$$

The time $t_{1}$ remains constant also in this case, if we consider the successive stages of movement. Relations (13) - (14) represent the principle of superposition of successive motions in their description in the form of Lagrange, which consists in replacing the Lagrange variables of the subsequent motion with the Euler variables of the preceding motion.

The sequence of implementation of each of the movements that form the corresponding composite motion as a result of superposition can be determined by specific time parameters, such as the angle of rotation $\varphi(t)$ and linear displacements $\boldsymbol{u}(\boldsymbol{t})$ during joint torsion and translational motion

$$
x_{i}=x_{i}\left(\alpha_{p}, \varphi(t), u(t)\right) \quad \text { at } \quad 0 \leq t \leq t_{2}
$$

or various components of the movement $u_{i}(t)$ along the coordinate axes during spatial translational motion [4-5].

The considered principle is also applicable to the simultaneous flow of combined movements, since the intervals of stages can be infinitely small and at each of them the movements from both types of movement will be summed up.

The form of the resulting motion (14) depends on the Lagrange coordinates of which of the motions will be replaced by Euler variables. For the sake of definiteness, in the future we will call nested or internal motions $x_{i}^{i n}=x_{i}^{i n}\left(\alpha_{p}, t\right)$, which replace the Lagrangian coordinates of another motion. The movement $x_{i}^{e x}=x_{i}^{e x}\left(\alpha_{p}, t\right)$ in which this replacement occurred, respectively, will be called superimposed or external. External and internal movements are similar to portable and relative ones in classical mechanics, but differ in the mandatory use of a single coordinate system. 
If the Euler and Lagrange variables coincide in the initial state $x_{i}\left(\alpha_{p}, t=0\right)=\alpha_{i}$, the superposition of motions is reduced to replacing the Lagrange variables of external motion $x_{i}^{e x}=x_{i}^{e x}\left(\alpha_{p}, t\right)$ with expressions for the corresponding Euler variables of internal motion $x_{i}^{i n}=x_{i}^{i n}\left(\alpha_{p}, t\right)$. The equations of combined motion $x_{i}=x_{i}\left(\alpha_{p}, t\right)$ in their sequential or simultaneous flow coincide with the equations of external motion after replacing the Lagrange variables with the corresponding equations for the Euler variables of internal motion

$$
x_{i}\left(\alpha_{p}, t\right)=x_{i}^{e x}\left(x_{i}^{i n}\left(\alpha_{p}, t\right), t\right)
$$

The difference between nested and superimposed motions can be insignificant when they are equivalent, for example, when rotating relative to two axes, or in processes of deformation, when the displacements from each type of deformation are insignificant compared to the initial coordinates of the particles. The number of movements involved in the superposition can be arbitrary.

The proposed principle does not contradict the generally accepted one and its consequence is the vector addition of both the velocities and accelerations of the superimposing movements at each moment of time.

Taking into account the general rules of differentiation of implicitly given functions and the geometric meaning of Lagrange variables $\left(\alpha=x^{i n}, \beta=y^{i n}, \gamma=z^{i n}\right.$ ) for the velocity components of the combined motion (15) we obtain

$$
x_{t}=x_{t}+x_{\alpha} x_{t}^{i n}+x_{\beta} y_{t}^{i n}+x_{\gamma} z_{t}^{i n}
$$

or, taking into account the rule of summation by repeated index, for any components

$$
x_{i, t}=x_{i, t}^{e x}+x_{i, p}^{e x} x_{p, t}^{i n}
$$

where the first term and the left multiplier refer to the portable (superimposed) movement, while the right multiplier is determined by the nested movement.

The components of the velocity of the external motion (the first terms) must be determined at any moment in time at the point in the observer's space that the particle will actually occupy, taking into account the two considered motions. Relations (16) can be interpreted geometrically as a vector sum [7] of the velocities of nested and superimposed motions, as can be seen in concrete examples with different types of motions. 
The equations for the acceleration components are not given, but the validity of the general statement about geometric addition is not in doubt, since the derivative of the velocity with respect to the scalar argument (time) does not change the rank of the tensor [7].

\section{Examples of superposition of motions for absolutely rigid bodies}

To write down the equations of motion in the form of Lagrange, it is enough to compare the position of the particles of the body at the initial and arbitrary moments of time. In particular, for the translational movement of a solid body along any spatial trajectory, the displacement projections for all particles must be the same

$$
x=x_{P}+\left(\alpha-\alpha_{P}\right), \quad y=\beta+y_{P}-\beta_{P}, \quad z=\gamma+z_{P}-\gamma_{P},
$$

matrix (2) takes the form

$$
x_{i, p} \equiv \frac{\partial x_{i}}{\partial \alpha_{p}}=\left[\begin{array}{lll}
1 & 0 & 0 \\
0 & 1 & 0 \\
0 & 0 & 1
\end{array}\right]
$$

Condition (18) can be considered a mathematical definition of translational motions of absolutely rigid bodies.

To write the equation of the plane-parallel motion of rotation of rigid body about a fixed pole in the plane $x-y$, consider the relative position of the pole of $P_{0}\left(\alpha_{P}, \beta_{P}\right)$ and an arbitrary point $M_{0}(\alpha, \beta)$, using the length $L_{0}$ and the slope of the straight line connecting these points, for the initial

$$
\alpha=\alpha_{P}+L_{0} \cos \varphi_{0}, \quad \beta=\beta_{P}+L_{0} \sin \varphi_{0}
$$

and the current time

$$
x=\alpha_{P}+L_{0} \cos \varphi=\alpha_{P}+L_{0} \cos \left(\varphi_{0}+\Delta \varphi\right), \quad y=\beta_{P}+L_{0} \sin \left(\varphi_{0}+\Delta \varphi\right),
$$

where $\Delta \varphi$ is the rotation angle of the body. Excluding from the last equations the length $L_{0}$ and the initial value of the angle $\varphi_{0}$ using equations (19), we obtain the system (the $z$ coordinate does not change)

$$
x=\alpha_{P}+\left(\alpha-\alpha_{P}\right) \cos \Delta \varphi-\left(\beta-\beta_{P}\right) \sin \Delta \varphi, \quad y=\beta_{P}+\left(\alpha-\alpha_{P}\right) \sin \Delta \varphi+\left(\beta-\beta_{P}\right) \cos \Delta \varphi, \quad z=\gamma
$$

From here, equations for rotation with respect to the origin can be obtained

$$
x=\alpha \cos \Delta \varphi-\beta \sin \Delta \varphi, \quad y=\alpha \sin \Delta \varphi+\beta \cos \Delta \varphi, \quad z=\gamma .
$$

Superposition of rotation (21) and translational displacement (17) in the form

$$
x=\alpha+x_{P}-\alpha_{P}, \quad y=\beta+y_{P}-\beta_{P}, \quad z=\gamma .
$$


allows us to obtain equations for two types of motion. If we consider the motion (21) to be external, we obtain the equations of translational motion of a body along a wheel rotating relative to the origin,

$$
x=\left(x_{P}-\alpha_{P}\right) \cos \Delta \varphi-\left(y_{P}-\beta_{P}\right) \sin \Delta \varphi, \quad y=\left(x_{P}-\alpha_{P}\right) \sin \Delta \varphi+\left(y_{P}-\beta_{P}\right) \cos \Delta \varphi, \quad z=\gamma
$$

If we consider the motion (22) to be external, then the equations will correspond to the rotation of a solid body relative to a moving pole

$$
x=x_{P}+\left(\alpha-\alpha_{P}\right) \cos \Delta \varphi-\left(\beta-\beta_{P}\right) \sin \Delta \varphi, \quad y=y_{P}+\left(\alpha-\alpha_{P}\right) \sin \Delta \varphi+\left(\beta-\beta_{P}\right) \cos \Delta \varphi, \quad z=\gamma .
$$

In these equations, the coordinates of the pole $x_{P}, y_{P}$ and the angle of rotation of the body $\Delta \varphi(t)$ depend on time. If they are differentiated by time, we obtain the relations between the velocities of the pole and an arbitrary particle, in particular for the system (23)

$$
d x / d t \equiv x_{t}=\left(x_{t}\right)_{P}-\varphi_{t}\left(y-y_{P}\right), \quad d y / d t \equiv y_{t}=\left(y_{t}\right)_{P}+\varphi_{t}\left(x-x_{P}\right), \quad d z / d t=0
$$

Equations (21)-(24) are sufficient to describe any motion of absolutely rigid bodies. For plane-parallel movements in other planes, they can be obtained by circular substitution, and for more complex spatial movements due to the superposition principle, which allows us to consider spatial processes as the simultaneous implementation of several plane-parallel movements [6]. In particular, for rotation around the $x$ and $y$ axes, taking into account (23) and replacing the designation of the rotation angles, we obtain

$$
\begin{aligned}
& x=\alpha, \quad y=y_{P}+\left(\beta-\beta_{P}\right) \cos \Delta \psi-\left(\gamma-\gamma_{P}\right) \sin \Delta \psi, \quad z=z_{P}+\left(\beta-\beta_{P}\right) \sin \Delta \psi+\left(\gamma-\gamma_{P}\right) \cos \Delta \psi, \\
& x=x_{P}+\left(\alpha-\alpha_{P}\right) \cos \Delta \xi+\left(\gamma-\gamma_{P}\right) \sin \Delta \xi, \quad y=\beta, \quad z=z_{P}-\left(\alpha-\alpha_{P}\right) \sin \Delta \xi+\left(\gamma-\gamma_{P}\right) \cos \Delta \xi .
\end{aligned}
$$

Matrix (2) for system (23) takes the form typical for rotations

$$
x_{i, p}=\left(\begin{array}{ccc}
\cos \Delta \varphi & -\sin \Delta \varphi & 0 \\
\sin \Delta \varphi & \cos \Delta \varphi & 0 \\
0 & 0 & 1
\end{array}\right)
$$

If two motions in the $x-y$ plane are rotational with respect to parallel axes, for example, a nested rotational with respect to a point with coordinates $(c, 0)$

$$
x=c+(\alpha-c) \cos \omega_{1} t-\beta \sin \omega_{1} t, \quad y=(\alpha-c) \sin \omega_{1} t+\beta \cos \omega_{1} t, \quad z=\gamma
$$

and a portable rotational with respect to the origin

$$
x=\alpha \cos \omega_{0} t-\beta \sin \omega_{0} t, \quad y=\alpha \sin \omega_{0} t+\beta \cos \omega_{0} t, \quad z=\gamma,
$$

as a result, we obtain a combined rotational motion 


$$
\begin{gathered}
x=c \cos \left(\omega_{0} t\right)+(\alpha-c) \cos \left[\left(\omega_{0}+\omega_{1}\right) t\right]-\beta \sin \left[\left(\omega_{0}+\omega_{1}\right) t\right] \\
y=c \sin \left(\omega_{0} t\right)+(\alpha-c) \sin \left[\left(\omega_{0}+\omega_{1}\right) t\right]+\beta \cos \left[\left(\omega_{0}+\omega_{1}\right) t\right], \quad z=\gamma
\end{gathered}
$$

with velocity components

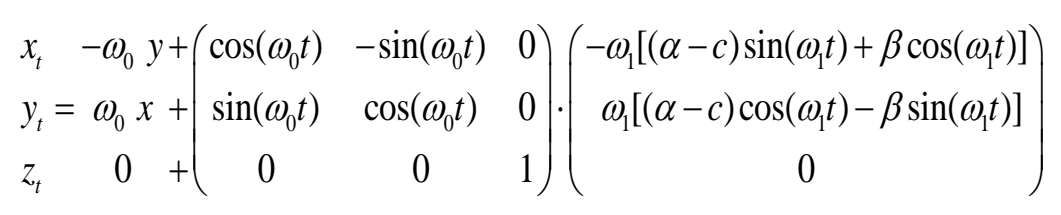

or, after multiplying the matrices,

$$
\begin{gathered}
x_{t}=-\omega_{0} y-\omega_{1}\left\{(\alpha-c) \sin \left[\left(\omega_{0}+\omega_{1}\right) t\right]+\beta \cos \left[\left(\omega_{0}+\omega_{1}\right) t\right]\right\}=-\omega_{0} y-\omega_{1}\left[y-c \sin \left(\omega_{0} t\right)\right], \\
y_{t}=\omega_{0} x+\omega_{1}\left\{(\alpha-c) \cos \left[\left(\omega_{0}+\omega_{1}\right) t\right]-\beta \sin \left[\left(\omega_{0}+\omega_{1}\right) t\right]\right\}=\omega_{0} x+\omega_{1}\left[x-c \cos \left(\omega_{0} t\right)\right], \quad z_{t}=0 .
\end{gathered}
$$

The equation for acceleration can be obtained by repeated differentiation of the components of the velocity. In all the cases considered, the rule of algebraic summation of the same-name projections of velocities and accelerations for nested and portable motions at each moment of time is fulfilled.

If a body rotating around the $z$ axis in accordance with the system (21) receives an additional rotation around the $x_{1}$ axis passing through the movable pole $P$ parallel to the $x$ axis of the observer's coordinate system in accordance with the system (25), then to describe the joint rotation, it is sufficient to substitute the right part for the Eulerian coordinates $x$ and $y$ from equations (21) instead of the Lagrangian coordinates $\alpha$ and $\beta$ in equations (25). As a result, we get

$$
\begin{gathered}
x=\alpha \cos \Delta \varphi-\beta \sin \Delta \varphi, \quad y=y_{P}+\left[(\alpha \sin \Delta \varphi+\beta \cos \Delta \varphi)-\beta_{P}\right] \cos \Delta \psi-\left(\gamma-\gamma_{P}\right) \sin \Delta \psi, \\
z=z_{P}+\left[(\alpha \sin \Delta \varphi+\beta \cos \Delta \varphi)-\beta_{P}\right] \sin \Delta \psi+\left(\gamma-\gamma_{P}\right) \cos \Delta \psi .
\end{gathered}
$$

If the rotation (25) occurs relative to the $x$-axis of the observer's coordinate system, then $y_{P}=z_{P}=\beta_{P}=\gamma_{P}=0$ and instead of the previous system, we obtain the equations of combined rotation (simultaneously or sequentially determine the time dependences of the angles $\Delta \varphi(t)$ and $\Delta \psi(t)$ )

$$
\begin{gathered}
x=\alpha \cos \Delta \varphi-\beta \sin \Delta \varphi, \quad y=(\alpha \sin \Delta \varphi+\beta \cos \Delta \varphi) \cos \Delta \psi-\gamma \sin \Delta \psi, \\
z=(\alpha \sin \Delta \varphi+\beta \cos \Delta \varphi) \sin \Delta \psi+\gamma \cos \Delta \psi .
\end{gathered}
$$


In the case under consideration, the rotations relative to the $z$ and $x$ axes of the observer's coordinate system become equivalent and any of them can be taken as external. If we take the external rotation (21), and the internal rotation (25) at $y_{P}=z_{P}=\beta_{P}=\gamma_{P}=0$, then as a result of the superposition, instead of (27) we get a new system of equations

$$
\begin{gathered}
x=\alpha \cos \Delta \varphi-(\beta \cos \Delta \psi-\gamma \sin \Delta \psi) \sin \Delta \varphi, \\
y=\alpha \sin \Delta \varphi+(\beta \cos \Delta \psi-\gamma \sin \Delta \psi) \cos \Delta \varphi, \quad z=\beta \sin \Delta \psi+\gamma \cos \Delta \psi
\end{gathered}
$$

However, the numerical results of calculations with the coincidence of the corresponding coordinates of the particles and the angles of rotation of the body relative to both axes will be the same. If one of the rotations is stopped, for example, take $\Delta \varphi=0$, both systems will coincide.

The composite motion (27) can be additionally superimposed with the rotation of the body around the $y$ axis, replacing the Lagrangian coordinates $\alpha_{p}$ in equations (26) with the corresponding expressions for the Eulerian coordinates $x_{i}$ from equations (27). Get the resulting motion

$$
\begin{gathered}
x=(\alpha \cos \Delta \varphi-\beta \sin \Delta \varphi) \cos \Delta \xi+[(\alpha \sin \Delta \varphi+\beta \cos \Delta \varphi) \sin \Delta \psi+\gamma \cos \Delta \psi] \sin \Delta \xi \\
y=(\alpha \sin \Delta \varphi+\beta \cos \Delta \varphi) \cos \Delta \psi-\gamma \sin \Delta \psi \\
z=-(\alpha \cos \Delta \varphi-\beta \sin \Delta \varphi) \sin \Delta \xi+[(\alpha \sin \Delta \varphi+\beta \cos \Delta \varphi) \sin \Delta \psi+\gamma \cos \Delta \psi] \cos \Delta \xi
\end{gathered}
$$

The correctness of the record in all cases of motion of absolutely solid bodies can be checked by fulfilling the condition of constancy of the volume of particles and the absence of deformation (9). For example, when moving in accordance with equations (28), the matrix of derivatives (2) takes the form

$$
x_{i, p}=\left[\begin{array}{ccc}
\sin \Delta \varphi \sin \Delta \psi \sin \Delta \xi+\cos \Delta \varphi \sin \Delta \xi & \cos \Delta \varphi \sin \Delta \psi \sin \Delta \xi-\sin \Delta \varphi \cos \Delta \xi & \cos \Delta \psi \sin \Delta \xi \\
\sin \Delta \varphi \cos \Delta \psi & \cos \Delta \varphi \cos \Delta \psi & -\sin \Delta \psi \\
\sin \Delta \varphi \sin \Delta \psi \cos \Delta \xi-\cos \Delta \varphi \sin \Delta \xi & \cos \Delta \varphi \sin \Delta \psi \cos \Delta \xi+\sin \Delta \varphi \sin \Delta \xi & \cos \Delta \psi \cos \Delta \xi
\end{array}\right]
$$

As in other cases, conditions (9) are met, the lengths of edges and particle volume do not change, and rotation occurs without deformation of the particles.

\section{Superposition of movions as a stage of dynamic analysis of mechanisms}

The superposition of plane-parallel motions makes it possible to describe the spatial motion of particles of links of hinge-lever, cam and other types of mechanisms of any complexity [6,8], for example, presented in Figures 1 and 2 , for subsequent dynamic analysis. 
Link 1 of the robot, the block diagram of which is shown in Figure 1, is fixed to the rack using a rotational kinematic pair, which allows the link to rotate relative to the $\mathrm{z}$ axis passing through the origin. The particles of the link change their position in the observer's space in accordance with equations (21), which, taking into account the accepted coordinate system and the position of the pole $P$, take the form

$$
x=\alpha \cos \Delta \theta_{z}-\beta \sin \Delta \theta_{z}, \quad y=\alpha \sin \Delta \theta_{z}+\beta \cos \Delta \theta_{z}, \quad z=\gamma
$$

Link 2 rotates relative to the y1 axis, using the drive in a kinematic pair with the point $A\left(\alpha_{A}=0,0, \gamma_{A}=H\right)$, in accordance with equations (26)

$$
\begin{gathered}
x=\alpha_{A}+\left(\alpha-\alpha_{A}\right) \cos \Delta \theta_{y}+\left(\gamma-\gamma_{A}\right) \sin \Delta \theta_{y}, \quad y=\beta, \\
z=\gamma_{A}+\left(\gamma-\gamma_{A}\right) \cos \Delta \theta_{y}-\left(\alpha-\alpha_{A}\right) \sin \Delta \theta_{y} .
\end{gathered}
$$

Links 2 and 3 are connected by a translational kinematic pair at point $B\left(\alpha_{B}=L 1,0, \gamma_{B}=H\right)$, the drive provides translational movement of link 3 relative to link 2 along the $x$ axis, in accordance with equations (4), for example, due to rack and pinion transmission

$$
x=\alpha+\left(x_{B}-\alpha_{B}\right)=\alpha+u(t), \quad y=\beta, \quad z=\gamma
$$

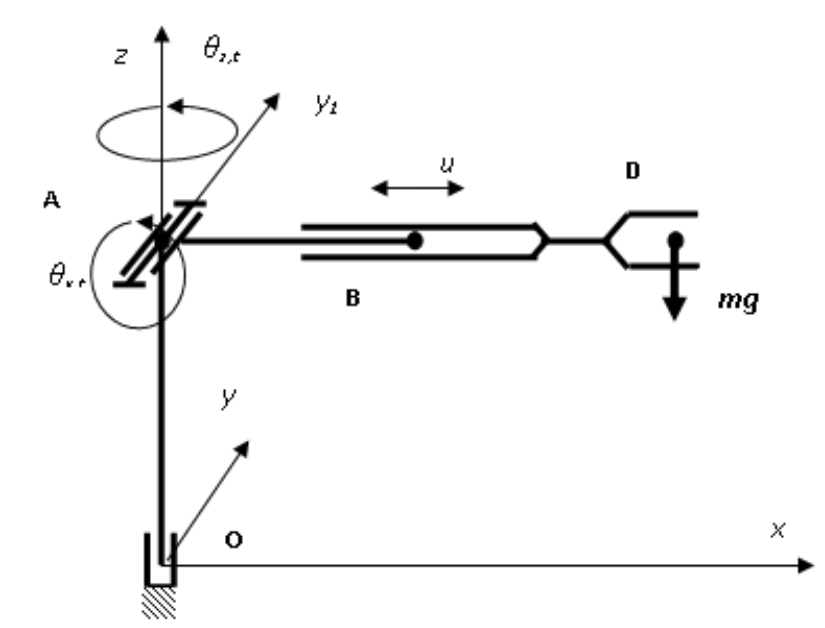

Figure 1. Block diagram of a three-link robot with two rotational and one translational kinematic pairs in the initial position

In accordance with rule (15), for the superpositions internal motion of link 2 with the external motion of link 1 , the Lagrange variables in equations (29) should be replaced by the corresponding equations from the system (30)

$$
(x)_{2}=\left[\alpha_{A}+\left(\alpha-\alpha_{A}\right) \cos \Delta \theta_{y}+\left(\gamma-\gamma_{A}\right) \sin \Delta \theta_{y}\right] \cos \Delta \theta_{z}-\beta \sin \Delta \theta_{z}
$$




$$
\begin{gathered}
(y)_{2}=\left[\alpha_{A}+\left(\alpha-\alpha_{A}\right) \cos \Delta \theta_{y}+\left(\gamma-\gamma_{A}\right) \sin \Delta \theta_{y}\right] \sin \Delta \theta_{z}+\beta \cos \Delta \theta_{z}, \\
(z)_{2}=\gamma_{A}+\left(\gamma-\gamma_{A}\right) \cos \Delta \theta_{y}-\left(\alpha-\alpha_{A}\right) \sin \Delta \theta_{y} .
\end{gathered}
$$

The subscript after the bracket specifies the number of the link for which they are applicable in the combined movement.

Using rule (15) again, we obtain the equations of motion for link 3, taking into account the internal motion (31) and the external one (32)

$$
\begin{gathered}
(x)_{3}=\left[\alpha_{A}+\left(\alpha+u-\alpha_{A}\right) \cos \Delta \theta_{y}+\left(\gamma-\gamma_{A}\right) \sin \Delta \theta_{y}\right] \cos \Delta \theta_{z}-\beta \sin \Delta \theta_{z} \\
(y)_{3}=\left[\alpha_{A}+\left(\alpha+u-\alpha_{A}\right) \cos \Delta \theta_{y}+\left(\gamma-\gamma_{A}\right) \sin \Delta \theta_{y}\right] \sin \Delta \theta_{z}+\beta \cos \Delta \theta_{z}, \\
(z)_{3}=\gamma_{A}+\left(\gamma-\gamma_{A}\right) \cos \Delta \theta_{y}-\left(\alpha+u-\alpha_{A}\right) \sin \Delta \theta_{y} .
\end{gathered}
$$

The block diagram, initial position and coordinate system for the second mechanism are shown in Fig. 2 . The $z$ axis is aligned with the axis of the vertical rack, along which the link 1 can move due to the drive at point $A(0,0, H)$. At the point $B$ of the link 1 with the initial coordinates $\alpha=L_{B}, \beta=0, \gamma=H$ a drive is fixed that provides rotational movement of the link 2 relative to the vertical axis $z_{1}$ with an angular velocity $\theta_{z 1, t}$. Links 2 and 3 are connected by a rotational kinematic pair $D\left(L_{D}, 0, H\right)$ with the possibility of rotation, due to an additional drive in this kinematic pair on link 2, relative to the vertical axis $z_{2}$ with an angular velocity $\theta_{z 2, t}$.

Independent motions from the initial state describe the equations:

link 1 with a pole at the origin by analogy with (17):

$$
x=\alpha, \quad y=\beta, \quad z=\gamma+w(t),
$$

link 2 with pole $B(L B, 0, H)$ by analogy with (20):

$$
x=L_{B}+\left(\alpha-L_{B}\right) \cos \Delta \theta_{z 1}-\beta \sin \Delta \theta_{z 1}, \quad y=\left(\alpha-L_{B}\right) \sin \Delta \theta_{z 1}+\beta \cos \Delta \theta_{z 1}, \quad z=\gamma,
$$

link 3, with the pole $D\left(L_{D}, 0, H\right)$ :

$$
x=L_{D}+\left(\alpha-L_{D}\right) \cos \Delta \theta_{z 2}-\beta \sin \Delta \theta_{z 2}, \quad y=\left(\alpha-L_{D}\right) \sin \Delta \theta_{z 2}+\beta \cos \Delta \theta_{z 2}, \quad z=\gamma
$$

where $\Delta \theta_{z 1}$ and $\Delta \theta_{z 2}$ are the angles of rotation relative to the $z 1$ and $z 2$ axes passing through points $B$ and $D$ parallel to the $z$ axis. 


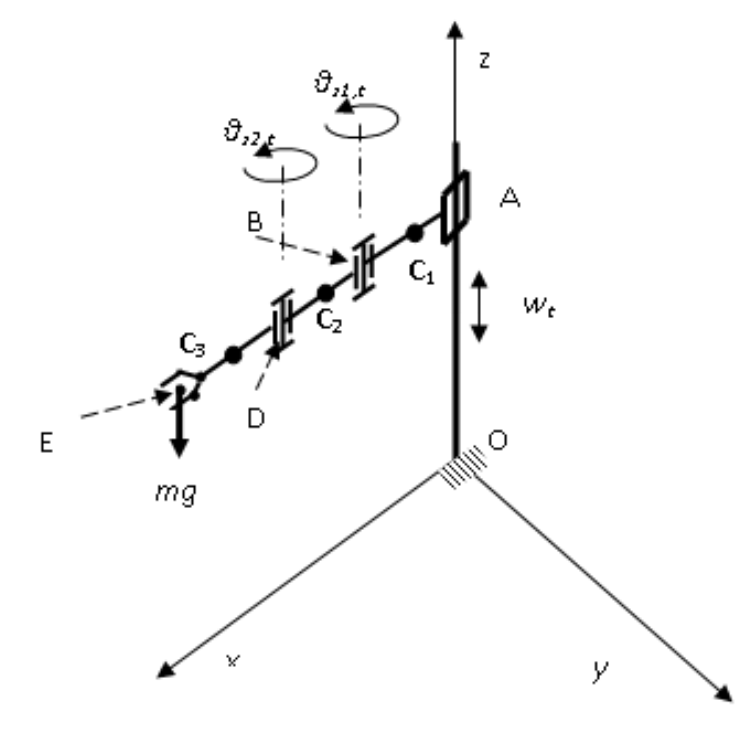

Figure 2. Coordinate system for a mechanism with 3 degrees of freedom

Taking the motion of link 1 as external and substituting the right-hand sides of the equations of system (34) instead of the Lagrangian coordinates of system (33), in accordance with equation (15) we obtain the equations of combined motion for link 2

$$
\begin{gathered}
(x)_{2}=L_{B}+\left(\alpha-L_{B}\right) \cos \Delta \theta_{z 1}-\beta \sin \Delta \theta_{z 1} \\
(y)_{2}=\left(\alpha-L_{B}\right) \sin \Delta \theta_{z 1}+\beta \cos \Delta \theta_{z 1}, \quad(z)_{2}=\gamma+w(t) .
\end{gathered}
$$

The combined motion (36) is external to the motion of link 3, so to determine the equations of motion of link 3, taking into account the motion of links 1 and 2, we replace the Lagrangian coordinates in system (36) with the corresponding expressions from system (35)

$$
\begin{gathered}
(x)_{3}=L_{B}+\left[b-L_{B}+\left(\alpha-L_{D}\right) \cos \Delta \theta_{z 2}-\beta \sin \Delta \theta_{z 2}\right] \cos \Delta \theta_{z 1}-\left[\left(\alpha-L_{D}\right) \sin \Delta \theta_{z 2}+\beta \cos \Delta \theta_{z 2}\right] \sin \Delta \theta_{z 1}, \\
(y)_{3}=\left[L_{D}-L_{B}+\left(\alpha-L_{D}\right) \cos \Delta \theta_{z 2}-\beta \sin \Delta \theta_{z 2}\right] \sin \Delta \theta_{z 1}+\left[\left(\alpha-L_{D}\right) \sin \Delta \theta_{z 2}+\beta \cos \Delta \theta_{z 2}\right] \cos \Delta \theta_{z 1}, \\
(z)_{3}=\gamma+w(t) .
\end{gathered}
$$

As noted above, the correctness of the record in all cases of motion of absolutely solid bodies can be checked by fulfilling the condition of constancy of the volume of particles and the absence of deformation (9).

The use of equations of the considered type for mechanisms of various complexity makes it possible to find forces in any cross-sections and kinematic pairs [7] that satisfy the law of conservation of energy at any time on any part of the mechanism from the energy balance 


$$
W_{e}=W_{k}+W_{p}
$$

where $W_{e}$ is the power of external forces, $W_{k}$ and $W_{p}$ are the rates of change in the kinetic and potential energy of the mechanical system under consideration. The kinetic energy for spatial motions can be determined by the Koenig formula

$$
E_{k}=0,5 m v_{C}^{2}+0,5\left(\theta_{x, t}^{2} J_{C}^{x}+\theta_{y, t}^{2} J_{C}^{y}+\theta_{z, t}^{2} J_{C}^{z}\right)
$$

where $J_{C}^{i}, \theta_{i, t}$ are the moments of inertia and angular velocities of rotation of the body relative to the axes specified in the indices. For given masses and moments of inertia of the bodies included in the system, the equations of motion (33), (36) and (37) uniquely determine the right side of the balance (38) for the mechanism, the block diagram of which is shown in Fig. 2. From the condition of equality of coefficients at linear or angular velocities in both parts of equation (38), we find the force factors $Q_{x}, Q_{y}, Q_{z}, M_{z 1}, M_{z 2}$ included in the left part necessary for performing the operations provided for by the mechanism. For example, for link 3, equation (38) takes the form

$$
\begin{aligned}
\left(Q_{x} x_{t}\right. & \left.+Q_{y} y_{t}+Q_{z} z_{t}\right)_{D}+\left(M_{z 1}\right)_{D} \theta_{z 1, t}+\left(M_{z 2}\right)_{D} \theta_{z 2, t}=m_{C 3}\left[x_{t} x_{t t}+y_{t} y_{t t}+\left(z_{t t}+g\right) z_{t}\right]_{C 3}+ \\
& +m_{E}\left[x_{t t} x_{t}+y_{t t} y_{t}+z_{t}\left(z_{t t}+g\right)\right]_{E}+\left(J_{C 3}^{z 1}+J_{E}^{z 1}\right) \theta_{z 1, t} \theta_{z 1, t t}+\left(J_{C 3}^{z 2}+J_{E}^{z 2}\right) \theta_{z 2, t} \theta_{z 2, t t}
\end{aligned}
$$

The lower indices indicate the point of the block diagram at which the masses $m_{K}$ and moments of inertia $J_{K}^{z i}$, as well as velocities and accelerations should be determined, taking into account the corresponding equations of combined motion, C3 - the center of mass of the link 3. For moments of inertia, the upper index indicates the axis relative to which the dynamic characteristic of the body should be indicated. The terms $\left(M_{z 1}\right)_{D} \theta_{z 1, t}$ and $\left(M_{z 2}\right)_{D} \theta_{z 2, t}$ in the left part of the balance (39) take into account the power spent on the rotation of the link 3 with angular velocities $\theta_{z 1, t}$ and $\theta_{z 2, t}$. The moment $\left(M_{z 2}\right)_{D}$ receives energy from the source at point $D$ and does not participate in further analysis. The power to rotate link 3 with angular velocity $\theta_{z 1, t}$ comes from the drive at point $B$ and therefore the moment $\left(M_{z 1}\right)_{D}$ must participate in the energy balance for link 2

$$
\begin{aligned}
& \left(Q_{x} x_{t}+Q_{y} y_{t}+Q_{z} z_{t}\right)_{B}+\left(M_{z 1}\right)_{B} \theta_{z 1, t}=m_{C 2}\left[x_{t} x_{t t}+y_{t} y_{t t}+z_{t}\left(z_{t t}+g\right)\right]_{C 2}+J_{C 2}^{z} \theta_{z 1, t} \theta_{z 1, t t}+ \\
& \quad+\left(Q_{x} x_{t}+Q_{y} y_{t}+Q_{z} z_{t}\right)_{D}+m_{D}\left[x_{t t} x_{t}+y_{t t} y_{t}+z_{t}\left(z_{t t}+g\right)\right]_{D}+2 \xi J_{D}^{z} \theta_{z 1, t} \theta_{z 1, t t} .
\end{aligned}
$$


Differentiating the system (37) twice in time and equating the coefficients in both parts of equality (39), for the power factors included in the left part, we obtain

$$
\begin{gathered}
\left(Q_{x}\right)_{D}=m_{C 3}\left(x_{t t}\right)_{C 3}+m_{E}\left(x_{t t}\right)_{E}, \quad\left(Q_{y}\right)_{D}=m_{C 3}\left(y_{t t}\right)_{C 3}+m_{E}\left(y_{t t}\right)_{E}, \quad\left(Q_{z}\right)_{D 2}=m_{C 3}\left[\left(z_{t t}\right)_{C 3}+g\right]+m_{E}\left[\left(z_{t t}\right)_{E}+g\right] \\
\left(M_{z 1}\right)_{D}=-m_{C 3}\left[\left(x_{t t}\right)_{C 3}\left(y_{C 3}-y_{D}\right)+\left(y_{t t}\right)_{C 3}\left(x_{C 3}-x_{D}\right)\right]+\left(J_{C 3}^{z}+J_{E}^{z}\right) \theta_{z 1, t} \theta_{z 1, t t} \\
\left(M_{z 2}\right)_{D}=\left(J_{C 3}^{z}+J_{E}^{z}\right) \theta_{z 2, t t}-m_{C 3}\left[\left(x_{t t}\right)_{C 3}\left(y_{C 3}-y_{D}\right)+\left(y_{t t}\right)_{C 3}\left(x_{C 3}-x_{D}\right)\right]-m_{E}\left[\left(x_{t t}\right)_{E}\left(y_{E}-y_{D}\right)+\left(y_{t t}\right)_{E}\left(x_{E}-x_{D}\right)\right]
\end{gathered}
$$

From the energy balance for link 2 we find

$$
\begin{aligned}
& \left(Q_{x}\right)_{B}=\left(Q_{x}\right)_{D}+m_{C 2}\left(x_{t t}\right)_{C 2}, \quad\left(Q_{y}\right)_{B}=\left(Q_{y}\right)_{D}+m_{C 2}\left(y_{t t}\right)_{C 2}, \quad\left(Q_{z}\right)_{B}=\left(Q_{z}\right)_{D}+m_{C 2}\left[\left(z_{t t}\right)_{C 2}+g\right]+m_{D}\left[\left(z_{t t}\right)_{D}+g\right] \\
& \left(M_{z 1}\right)_{B}=\left(J_{C 2}^{z}+J_{D}^{z}\right) \theta_{z 1, t t}-m_{C 2}\left(x_{t t}\right)_{C 2}\left(y_{C 2}-y_{B}\right)-m_{D}\left(x_{t t}\right)_{D}\left(y_{D}-y_{B}\right)+m_{C 3}\left(y_{t t}\right)_{C 2}\left(x_{C 2}-x_{B}\right)+m_{D}\left(y_{t t}\right)_{D}\left(x_{D}-x_{B}\right) .
\end{aligned}
$$

For link 1, which performs translational movement relative to the $\mathrm{z}$ axis, the energy balance includes the rate of change of potential and kinetic energy from translational motion, as well as the power of forces $\left(Q_{i}\right)_{K}$ from an external energy source at point A and transmitted to other links of the mechanism at point B

$$
\begin{aligned}
& \left(Q_{x} x_{t}+Q_{y} y_{t}+Q_{z} z_{t}\right)_{A}=m_{C 1}\left(x_{t} x_{t t}+y_{t} y_{t t}+z_{t} z_{t t}\right)_{C 1}+m_{C 1} g\left(z_{t}\right)_{C 1}+ \\
& +\left(Q_{x} x_{t}+Q_{y} y_{t}+Q_{z} z_{t}\right)_{B}+m_{B}\left(x_{t t} x_{t}+y_{t t} y_{t}+z_{t} z_{t t}\right)_{B}+m_{B} g\left(z_{t}\right)_{B}
\end{aligned}
$$

The forces at point A of link 1, taking into account the energy balance (40), are

$$
\left(Q_{x}\right)_{A}=\left(Q_{x}\right)_{B}+m_{B}\left(x_{t t}\right)_{B}+m_{C 1}\left(x_{t t}\right)_{C 1}, \quad\left(Q_{y}\right)_{A}=\left(Q_{y}\right)_{B}+m_{B}\left(y_{t t}\right)_{B}+m_{C 1}\left(y_{t t}\right)_{C 1}, \quad\left(Q_{z}\right)_{A}=\left(Q_{z}\right)_{B}+m_{B}\left(z_{t t}\right)_{B}+m_{C 1}\left(z_{t t}\right)_{C 1}+\left(m_{C 1}+m_{B}\right) g
$$

Dynamic analysis should start from the links that are most remote from external energy sources, then the force factors at point B will be calculated beforehand and equation (40) provides the determination of factors $\left(Q_{i}\right)_{A}$ at point A from the condition of equality of coefficients with the same velocity components in both parts of the energy balance for the considered link of the mechanism. The moments $\left(M_{x}\right)_{A}$ and $\left(M_{y}\right)_{A}$ in the support A due to the absence of angular velocities do not produce power and do not participate in the energy balance, they can be determined from the generally accepted equations of statics [1-3].

The energy balance of type (38) can be recorded for any part of the mechanism, including the entire structure from the rack to the working tool at point E. However, in this case, the power of the local drives, as well as the forces and moments in the kinematic pairs of the mechanism remain unknown. 
Taking into account that the principle of superposition in the space of Lagrange variables provides for the use of only one common reference system of the observer, such a description eliminates the problems of non-inertial coordinate systems.

\section{Superposition of motions for processes of deformation}

The superposition principle is applicable to both absolutely solid and deformable bodies, and in the latter case, taking into account small displacements, the choice of external and internal movements often (except for cases of steady-state deformation processes) does not matter. The resulting equations may take different forms, but the numerical results are almost identical. The characteristics of the deformed state at an arbitrary time interval can be found in terms of the derivatives $x_{i, p} \equiv \partial x_{i} / \partial \alpha_{p}$ of the combined motions according to the equations (3)-(8) [5-6].

If the characteristics of the deformed state are known at each stage, the product of the values of the corresponding invariants can be used for the ratio of volumes

$$
R_{02}=\frac{\delta V_{2}}{\delta V_{0}}=R_{01} R_{12}=\left|\begin{array}{ccc}
x_{\alpha} & x_{\beta} & x_{\gamma} \\
y_{\alpha} & y_{\beta} & y_{\gamma} \\
z_{\alpha} & z_{\beta} & z_{\gamma}
\end{array}\right|=\left|\begin{array}{ccc}
x_{\xi} & x_{\eta} & x_{\zeta} \\
y_{\xi} & y_{\eta} & y_{\zeta} \\
z_{\xi} & z_{\eta} & z_{\zeta}
\end{array}\right| \cdot\left|\begin{array}{ccc}
\xi_{\alpha} & \xi_{\beta} & \xi_{\gamma} \\
\eta_{\alpha} & \eta_{\beta} & \eta_{\gamma} \\
\zeta_{\alpha} & \zeta_{\beta} & \zeta_{\gamma}
\end{array}\right| .
$$

For other characteristics of the deformed state, the additivity condition can be used. In particular, for linear stretching with equations of motion

$$
x=\alpha \exp \left(\varepsilon_{x x}\right), \quad y=\beta \exp \left(-\mu \varepsilon_{x x}\right), \quad z=\gamma \exp \left(-\mu \varepsilon_{x x}\right)
$$

for the average deformations (19), we obtain for the individual stages $\varepsilon_{01}$ and $\varepsilon_{02}$

$$
e_{01}=1+\varepsilon_{01}(1-2 \mu) / 3+\varepsilon_{01}^{2}\left(1+2 \mu^{2}\right) / 6 ; \quad e_{12}=1+\varepsilon_{12}(1-2 \mu) / 3+\varepsilon_{12}^{2}\left(1+2 \mu^{2}\right) / 6
$$

and for the general time interval

$$
e_{02}=1+\varepsilon_{02}(1-2 \mu) / 3+\varepsilon_{02}^{2}\left(1+2 \mu^{2}\right) / 6
$$

Since each of these measures is close to 1 in magnitude, when determining their values over two stages, their increments should be added up

$$
e_{02}-1=\varepsilon_{02}(1-2 \mu) / 3+\varepsilon_{02}{ }^{2}\left(1+2 \mu^{2}\right) / 6 \approx\left(e_{01}-1\right)+\left(e_{12}-1\right) \approx\left(\varepsilon_{01}+\varepsilon_{12}\right)(1-2 \mu) / 3 \text {. }
$$

With the same accuracy, the additivity condition is applicable for standard deviations (5)

$$
\Gamma_{01}=\sqrt{2 / 3}\left[\varepsilon_{01}(1+\mu)+0,5 \varepsilon_{01}^{2}\left(1-\mu^{2}\right)\right], \quad \Gamma_{12}=\sqrt{2 / 3}\left[\varepsilon_{12}(1+\mu)+0,5 \varepsilon_{12}^{2}\left(1-\mu^{2}\right)\right] \text {, }
$$




$$
\Gamma_{02}=\sqrt{2 / 3}\left[\varepsilon_{02}(1+\mu)+0,5 \varepsilon_{02}^{2}\left(1-\mu^{2}\right)\right] \approx \Gamma_{01}+\Gamma_{12},
$$

and increments of the energy in the elastic deformation region (6)

$$
\begin{gathered}
\left(\Delta \Gamma_{e}^{2}\right)_{01}=2 \varepsilon_{01}(1-2 \mu)+2 \varepsilon_{01}^{2}\left(1+2 \mu^{2}\right), \quad\left(\Delta \Gamma_{e}^{2}\right)_{12}=2 \varepsilon_{12}(1-2 \mu)+2 \varepsilon_{12}^{2}\left(1+2 \mu^{2}\right), \\
\left(\Delta \Gamma_{e}^{2}\right)_{02}=2 \varepsilon_{02}(1-2 \mu)+2 \varepsilon_{02}^{2}\left(1+2 \mu^{2}\right) .
\end{gathered}
$$

One can also verify the validity of these statements in the field of elastic deformations by the example of joint stretching with equations (41) and twisting with equations

$$
x=\alpha, \quad y=\beta \cos \Delta \psi-\gamma \sin \Delta \psi, \quad z=\beta \sin \Delta \psi+\gamma \cos \Delta \psi,
$$

where $\Delta \psi=\theta \alpha / L, \theta$ is the angle of twisting of the rod on the length $L$. As a result of the superposition, we obtain equations for simultaneous or sequential pressing with torsion of a cylindrical rod

$$
x=\alpha \exp \left(\varepsilon_{x x}\right), \quad y=(\beta \cos \Delta \psi-\gamma \sin \Delta \psi) \exp \left(-\mu \varepsilon_{x x}\right), \quad z=(\beta \sin \Delta \psi+\gamma \cos \Delta \psi) \exp \left(-\mu \varepsilon_{x x}\right) \text {. }
$$

Taking into account the peculiarities of the processes under consideration, either Cartesian or cylindrical coordinate systems can be used. The main relations for calculating kinematic and energy parameters during the transition from a Cartesian system to a cylindrical one are given in [5-6].

For example, in the region of large plastic deformations for a homogeneous precipitation of a cylindrical sample (in the absence of friction on the contact surfaces), the equations of motion instead of (41) can be written as [5]

$$
\rho=\rho_{0} \sqrt{h_{0} / h}, \quad \quad \quad z=z_{0} h / h_{0}, \quad \varphi=\varphi_{0} .
$$

For a joint pressing with torsion, instead of (42), it is advisable to use the equations for torsion

$$
\rho=\rho_{0}, \quad \quad z=z_{0}, \quad \varphi=\varphi_{0}+(\theta / L) z_{0} .
$$

When choosing any of the motions (43) or (44) as external, we obtain the equations of combined pressing with torsion in the region of plastic deformations

$$
\rho=\rho_{0} \sqrt{h_{0} / h}, \quad z=z_{0} h / h_{0}, \quad \varphi=\varphi_{0}+(\theta / L) z_{0} .
$$

The volume of the workpiece does not change during deformation

$$
R=\frac{\delta V}{\delta V_{0}}=\frac{\rho}{\rho_{0}}\left|\begin{array}{ll}
\partial \rho / \partial \rho_{0} & \partial \rho / \partial z_{0} \\
\partial z / \partial \rho_{0} & \partial z / \partial z_{0}
\end{array}\right|=\sqrt{h_{0} / h}\left|\begin{array}{cc}
\sqrt{h_{0} / h} & 0 \\
0 & h / h_{0}
\end{array}\right|=1 .
$$

As noted above, uniform deformation provides sufficient conditions to meet the equilibrium conditions. In cases of inhomogeneous deformation, such as bending, additional verification of the energy conservation law is required. 
For the combined bending of a wide strip with its stretching, you can use the equations for bending [5]

$$
x=\sin (\alpha / r) \sqrt{r(r+2 \beta)}, \quad y=\cos (\alpha / r) \sqrt{r(r+2 \beta)}-r, \quad z=\gamma
$$

where $r$ is the radius of curvature of the layer with the coordinate $\beta=\left.y\right|_{t=0}=0$, and for stretching under plane deformation conditions instead of (41)

$$
x=\alpha \exp \left(\varepsilon_{x}\right), \quad y=\beta \exp \left(-\varepsilon_{x}\right), \quad z=\gamma
$$

Taking the extension as an external motion, as a result of the superposition we get

$$
x=\exp \left(\varepsilon_{x}\right) \sqrt{r(r+2 \beta)} \sin (\alpha / r), \quad y=\exp \left(-\varepsilon_{x}\right)[\cos (\alpha / r) \sqrt{r(r+2 \beta)}-r], \quad z=\gamma
$$

If is considered a bend how the external motion, then the equations of combined motion will have a different form

$$
\begin{gathered}
x=\sin \left[\alpha \exp \left(\varepsilon_{x}\right) / r\right] \sqrt{r\left[r+2 \beta \exp \left(-\varepsilon_{x}\right)\right]}, \\
y=\cos \left[\alpha \exp \left(\varepsilon_{x}\right) / r\right] \sqrt{r\left[r+2 \beta \exp \left(-\varepsilon_{x}\right)\right]}-r, \quad z=\gamma,
\end{gathered}
$$

but the numerical results of the calculation for the two variants are almost identical due to the smallness of the $e_{x}$.

For steady-state processes with deformation, the external movement of the workpiece must be at a known tool speed. For example, cross-helical rolling can be considered as the superposition of translational motion with a constant velocity $\mathrm{v} 0$ in the direction of the rolling axis $\mathrm{z}$

$$
x=\alpha, \quad y=\beta, \quad z=\gamma+v_{0} t
$$

rotations with angular velocity $\omega_{0}$ around the $z$ axis

$$
x=\alpha \cos \left(\omega_{0} t\right)-\beta \sin \left(\omega_{0} t\right), \quad y=\alpha \sin \left(\omega_{0} t\right)+\beta \cos \left(\omega_{0} t\right), \quad z=\gamma
$$

and direct deformation in the space between the rolls and in the adjacent volumes (external deformation zones). Assuming the deformation of homogeneous and using a logarithmic measure

$$
\varepsilon=\ln \left(l / l_{0}\right)
$$

the process can be described by the simplest equations as in linear stretching in the direction of the $\mathrm{z}$ axis

$$
z=\gamma \exp \left(\varepsilon_{z}\right), \quad x=\alpha \exp \left(-0,5 \varepsilon_{z}\right), \quad y=\beta \exp \left(-0,5 \varepsilon_{z}\right)
$$

If the distance between the planes of the beginning and end of the deformation is denoted by $\mathrm{L}$, then we can write $\varepsilon_{z}=\varepsilon_{k} v_{c} t / L$, where $\varepsilon_{k}$ is the deformation of the particles at the exit from the deformation center, $v_{c}$ is the 
workpiece velocity averaged over the length of the deformation center. As a result of the superposition, the equations of combined motion take the form

$$
\begin{gathered}
x=\exp \left(-\varepsilon_{0} v_{c} t / L\right)\left[\alpha \cos \left(\omega_{0} t\right)-\beta \sin \left(\omega_{0} t\right)\right], \\
y=\exp \left(-\varepsilon_{0} v_{c} t / L\right)\left[\alpha \sin \left(\omega_{0} t\right)+\beta \cos \left(\omega_{0} t\right)\right], \quad z=\gamma \exp \left(-\varepsilon_{0} v_{c} t / L\right)+v_{0} t .
\end{gathered}
$$

The Lagrangian coordinates $\alpha$ and $\beta$ correspond to the coordinates of the particles in the plane of the beginning of the deformation, the coordinate $\gamma$ is the value of $z$ at $t=0$. The deformation occurs without changing the volume of the particles. In the absence of deformation $(\varepsilon k=0)$, the particle velocity in the $z$-axis direction remains unchanged. The differentiation of these equations allows us to determine the components of velocity, deformation and other kinematic, and then the force and energy local and integral characteristics necessary for calculating the energy-force parameters or optimizing the process.

Only geometric features by the superposition of equations of motion (volume change, aspect ratios of infinitesimal particles, and other deformation features) are considered above. However, any kind of motion must meet the requirements of the law of conservation of energy. For absolutely solid bodies, this is usually reduced to determining the forces at the contact of the links of the mechanism [8]. For processes with deformation, energy conservation allows us to explain the energy features of free vibrations and resonance [9-10].

This research received no external funding.

The funders had no role in the design of the study; in the collection, analyses, or interpretation of data; in the writing of the manuscript, or in the decision to publish the results.

Author ID in ORCID ～http://orcid.org/0000-0001-9977-3342

\section{References}

1. Prager V. Introduction to the mechanics of continuous media. - Moscow: Foreign Literature, 1963. - 312 p.

2. Sedov L. I. Mechanics of a continuous medium. Vol. 1. - Moscow: Nauka, 1970. - 492 p.

3. Rabotnov Yu. N. Mechanics of a deformable solid. - Moscow: Nauka, 1979. - 744 p.

4. Alyushin Yu. A. The principle of superposition of motions in the space of Lagrange variables //Problems of mechanical engineering and reliability of machines. -2001. - No. 3, pp. 13-19.

5. Alyushin Yu. A. Solid mechanics in Lagrange variables. - Moscow: Mashinostroenie, 2012. -192 p.

6. Alyushin Yu. A. Energy fundamentals of mechanics. - Lambert Academic Publishing, 2016. - 281 p.

7. Korn G., Korn T. Handbook of mathematics for scientists and engineers. - M.: Nauka, 1986. - 720 p. 
8. Alyushin Yu. A., Verzhansky P. M. Structural, kinematic and dynamic analysis of lever mechanisms. - Ed. Dom MISIS, 2015. $104 \mathrm{p}$.

9. Alyushin Yu. A. Energy features of free vibrations in elastic bodies // Physical Mesomechanics. Vol. 22. - 2019. - No. 3, pp. 77-87. DOI: 10.24411/1683-805X-2018-13007.

10. Alyushin Yu. A. Energy based resonance in elastic bodies // Physical Mesomechanics. Vol. 22. - 2019. - No. 5, pp. 42 - 53. DOI: 10.24411/1683-805X-2019-15006. 